\title{
Cruzando fronteras en vulnerabilidad. Estudio de la protección social transnacional en el Sur Global
}

Recibido: 4 de mayo de 2020 Revisado: 3 de agosto de 2020 Aprobado: 31 de agosto de 2020

Koen Voorend Holandés. PhD, profesor catedrático de la Universidad de Costa Rica: investigador del Instituto de Investigaciones Sociales, docente de la Escuela de

Ciencias de la Comunicación

Colectiva y del posgrado de

Comunicación y Desarrollo. Intereses de investigación en temas de política social, derechos sociales, migraciones y desigualdad. Correo electrónico: koen.voorend@ucr.ac.cr

Daniel José Alvarado Abarca Costarricense. Licenciado en Ciencias Políticas y bachiller en Sociología de la Universidad de Costa Rica. Intereses de investigación en temas de narcotráfico, seguridad regional e integración centroamericana migración y política social. Correo electrónico: daniel.alvarado.abarca18@gmail.

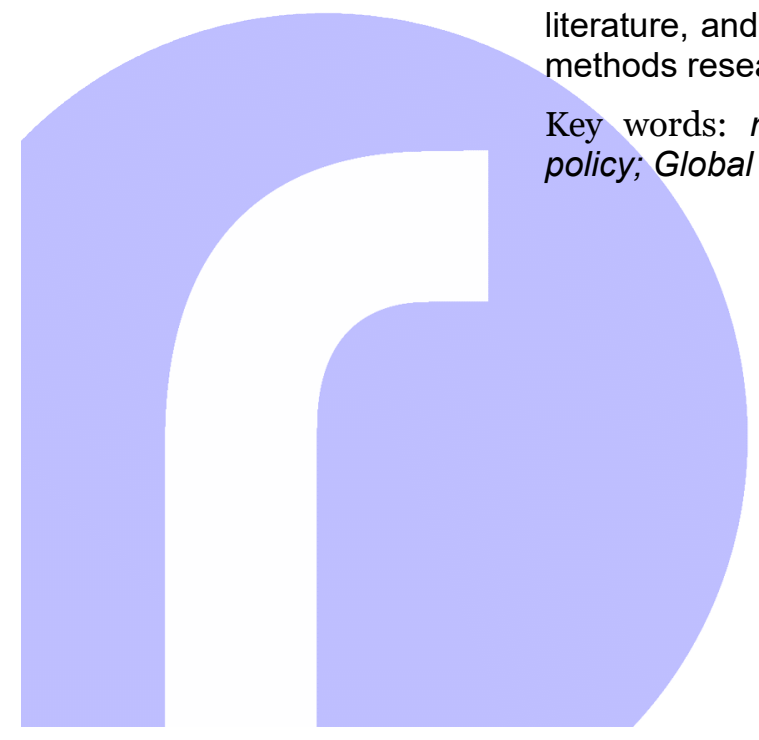

https://revistas.uned.ac.cr/index.php/rupturas (c) (1) (3) (-)
Resumen: Más de 270 millones de personas en el mundo viven en países diferentes al de su nacimiento. Estas acceden a servicios sociales, cuidan a miembros de sus familias, ahorran para su jubilación y opinan en la agenda pública. Sin embargo, se conoce relativamente poco sobre las prácticas de protección social de estas personas migrantes. En este artículo argumentamos que urge una agenda académica para indagar sobre las prácticas de protección social transnacional de migrantes, especialmente en el Sur global que esclarezca la capacidad de lidiar con los riesgos sociales de la vida cruzando fronteras nacionales. Para ello, discutimos lecciones claves de diferentes cuerpos de literatura y proponemos un marco analítico que se fundamenta en la investigación cuantitativa y cualitativa.

Palabras clave: migración; protección social transnacional; riesgos sociales; política social; Sur global

\section{Crossing National Borders in Vulnerability. The Study of Transnational Social Protection in the Global South}

Abstract: More than 270 million people live in countries other than that of their birth. These people access social services, take care of family members, save money for their pensions and contribute to public debates. However, we know little of how these people organize their social protection: how do they deal with social risks as migrants moving across borders? In this article, we argue for the necessity of an academic agenda on transnational social protection, especially in the Global South. This agenda should focus on migrants' capacity of dealing with social risks on both sides of national borders. For that purpose, we discuss key lessons from different bodies of literature, and we propose an analytical framework while arguing for a mixedmethods research agenda.

Key words: migration; transnational social protection; social risks; social policy; Global South. 


\section{Introducción}

¿Cómo y quién protege al migrante hondureño que salió de San Pedro Sula a sus 29 años de vida, con su hijo de seis meses, en búsqueda de escapar de la violencia que vive en su vecindario desde que tiene memoria? ¿Cuál es el nivel de protección social formal de una migrante nicaragüense no documentada en San José, Costa Rica, quien carece de acceso a los servicios de salud pública costarricense, pero espera a su hijo a la salida de la escuela pública para llevarlo a él a una cita en el hospital público? O ¿cómo funciona la protección social informal de la trabajadora doméstica peruana en Argentina que manda remesas mensualmente a Lima para apoyar y mantener a su familia en los espacios donde los programas de bienestar del Estado peruano se quedan cortos?

De manera más general, ¿cuándo, por quién y cómo se protegen las personas en movimiento dentro y fuera del marco tradicional del Estado-nación? ¿Cuáles arreglos de protección social, formal e informal están surgiendo ante las dinámicas migratorias cambiantes?

Cada vez más, las personas eligen o son forzadas a llevar vidas que cruzan las fronteras nacionales. Para el año 2019, más de 270 millones de personas se reportaron viviendo en otro país diferente al de su nacimiento (Department of Economic and Social Affairs 2019). Estas personas acceden a servicios sociales, cuidan a miembros de sus familias, ahorran para su jubilación y opinan en la agenda pública en más de un Estado nación. Sus "hogares" se encuentran dispersados por diferentes partes del mundo, por un lado, en donde tienen sus familias, sus diásporas y, por otro, en donde desarrollan su vida laboral, estudiantil y personal.

Existe un cuerpo creciente de literatura sobre diversos aspectos de estas vidas transnacionales, especialmente en cuanto al cuidado familiar que estas personas ejercen, pero resulta necesario ahondar en dinámicas transnacionales más amplias desde el llamado "Sur". La protección social, entendida como las prácticas, políticas y programas que determinan la capacidad de lidiar con riesgos sociales, se ha considerado durante mucho tiempo como un asunto entre los Estados y sus ciudadanos. En los últimos veinte años, con la literatura de los regímenes de bienestar en América Latina, ha surgido un reconocimiento de que la protección social depende de una constelación de prácticas que van más allá del Estado, que incluyen también al mercado, a las familias, y a las comunidades. En ese sentido, se torna necesario profundizar el conocimiento sobre las personas migrantes y sus prácticas de protección social, que contienen un componente importante de transnacionalismo. Cada vez más personas dependen, para su capacidad de lidiar con los riesgos sociales de la vida, de prácticas de Estados, de mercados, del tercer sector (ONGS o Iglesias) y de sus redes sociales que trascienden las fronteras nacionales. 
En este artículo argumentamos que urge una agenda de investigación en América Latina sobre la protección social formal e informal de personas migrantes al incorporar de manera explícita y transversal un lente transnacional. Para fundamentar este argumento, se proponen dos objetivos principales. Primero, se ofrece un estado de la cuestión de la literatura sobre la protección social transnacional, en su mayoría, desarrollada desde Europa y literaturas afines como la de regímenes de bienestar, la literatura sobre migración internacional y protección social y la de familias transnacionales, en particular, del cuidado transnacional. Al repasar estas literaturas, sus aportes y vacíos, se discuten los conceptos de protección social y cómo se entiende el transnacionalismo en este contexto.

Segundo, se presenta una reflexión sobre lo que implica estudiar la protección social formal e informal en y desde el Sur global y el valor agregado de hacerlo con un lente transnacional. Se propone un marco analítico para estudiar la protección social transnacional y se ofrece una discusión sobre su estudio, tanto teórico como metodológico, en y desde el Sur global.

\section{Estudiando la protección social y migración (en América Latina)}

La protección social se entiende de manera general como las políticas, los programas, las personas, las organizaciones e instituciones que proveen y protegen a las personas (Levitt et al. 2016). Por un lado, incluye la política social, o lo que varios estudios denominan la protección social formal (o estatal) (Barglowski, Bilecen y Amelina 2015; Faist y Bilecen 2015; SabatesWheeler y Feldman 2011). Son todas aquellas intervenciones estatales. Normalmente, se financian de manera pública y las regulan formalmente los Estados (Bilecen y Barglowski 2015). Como objetivo se proponen evitar que las personas enfrenten la pérdida de ingresos y de oportunidades vitales. Además, buscan promover condiciones dignas de vida y de trabajo (Fischer 2010; Mkandawire 2005). Por otro lado, incorpora la protección social informal, que es proporcionada por las redes sociales basadas en normas colectivas como la solidaridad comunitaria, la reciprocidad, el altruismo y las obligaciones (Bilecen y Barglowski 2015). Existe un consenso en la literatura que estas formas de protección social, formal e informal, se cruzan, se complementan y se compensan para formar lo que Faist (2013) ha llamado ensamblajes de protección social.

Uno de los cuerpos de literatura más importantes para la discusión sobre la protección social de migrantes es el que estudia la protección social formal en América Latina. Esta se enfoca, principalmente, en la política social estatal que reconoce, sin embargo, los altos grados de estratificación e informalidad que caracterizan los regímenes de política social en la región. Se trata de la literatura sobre regímenes de bienestar en América Latina, que nace a partir del famoso trabajo de Esping-Andersen (1990) y adaptaciones posteriores 
(Rudra 2007; Orloff 1993). Los supuestos básicos para la literatura sobre Estados de bienestar en Europa -un Estado legítimo y redistributivo con niveles de protección social altos y mercados laborales formales desarrolladosno aplican para el llamado Sur (Rudra 2007) ni a América Latina en particular (Martínez-Franzoni 2008; Barrientos 2004) y esta literatura debe ser adaptada para tales efectos. Esto bajo el supuesto de que los regímenes de bienestar pueden o no ser estatales (Gough y Wood 2004) y son en su mayoría 'informales' (Barrientos 2004) y 'exclusivos' (Filgueira 2007) con mercados laborales, que no proveen suficiente empleo formal y con políticas públicas relativamente débiles (Gough y Wood 2004). Igualmente, esta literatura se alejó del concepto del "Estado de bienestar" -entendido como un Estado que garantiza un nivel mínimo de bienestar para la mayoría, sino para todos sus ciudadanos, proponiendo el concepto de régimen de bienestar, entendido como una constelación de prácticas de tres instituciones que producen bienestar: el mercado, el Estado y la familia (Martínez-Franzoni 2008).

Más recientemente, ha surgido un renovado interés en la política social y en particular en el universalismo, a partir de la expansión de los arreglos de bienestar en América Latina durante las últimas dos décadas. Hay un consenso en esta literatura de que hubo una expansión, pero no sobre los factores que la expliquen, ni sobre si esta expansión es de naturaleza transformativa, ni sobre la precisa definición de la expansión misma (Martínez-Franzoni y Sánchez-Ancochea 2016; Huber y Stephens 2012; Filgueira et al. 2011; Haggard y Kaufman 2008). En este debate, destaca la centralidad en las explicaciones de regímenes políticos y composiciones partidarias-electorales, trayectorias históricas y las condiciones fiscales (implícita o explícitamente vinculadas con el boom de los commodities) (Arza 2009).

Sin embargo, los análisis en la literatura de regímenes de bienestar y la expansión de la política social se caracterizan por una ausencia de migrantes. Además, se ha basado casi exclusivamente en los marcos nacionales en que se desenvuelven las prácticas estatales, mercantiles y familiares de protección social, sin pensar en posibles procesos transnacionales. A pesar de ello, de esta literatura se rescatan dos cosas muy importantes. Primero, el lente comparativo es importante para entender variaciones en los niveles, la calidad y las constelaciones de protección social que las personas enfrentan. Segundo, la conceptualización del bienestar se destaca como una capacidad de lidiar con riesgos sociales y que esta capacidad depende de una constelación de prácticas estatales, mercantiles y familiares.

El segundo cuerpo de literatura relevante es el de las migraciones internacionales, en particular el que estudia los marcos legales y los derechos sociales, por un lado y el que estudia el nexo entre la migración y la política social, por otro lado. Primero, en Europa, existe una literatura extensa y rica sobre el otorgamiento de derechos sociales a poblaciones inmigrantes, las implicaciones para la integración, diferentes regímenes de integración o incorporación ( Lucassen 2016; Papadopulos 2011; Castles y Miller 2009), las diferencias de bienestar entre poblaciones de migrantes y las repercusiones de la inmigra- 
ción sobre la estabilidad política y financiera de la política social (Joppke 2012; Wilkinson y Craig 2011; van Hooren 2011; Kivisto y Faist 2007; Hansen y Castles 2006; Baldwin-Edwards 2002; Kofman et al. 2000; Schierup, Bommes y Geddes 2000).

En América Latina, esta literatura ha tenido un desarrollo menor, pero de gran importancia, que ha surgido especialmente en países con importantes flujos de inmigración y con regímenes de política social más estables, como Argentina, Chile y Costa Rica. Muchos de estos estudios analizan el reconocimiento legal de los derechos (sociales) en las reformas migratorias (CELS 2013; Begala 2012; Novick 2012; Torres 2012; Ceriani 2011; Cerutti 2011; Domenech 2011; Courtis, Liguori y Cerutti 2010; Novick 2010; Carrasco 2008; Domenech 2008; Novick 2008; Courtis y Pacecca 2007; Domenech 2007; Jelin 2006; Asa y Ceriani 2005; Varela 2005; Giustiniani 2004; Oteiza y Novick 2000). Sin embargo, quedan aún pendientes respuestas a temas como la manera en que la migración podría afectar la política social en el Sur y las reacciones de los Estados ante la demanda de servicios sociales, sobre todo, si las poblaciones migrantes realmente se incorporan a estos servicios; es decir, el acceso real, con notables excepciones (Voorend 2019).

Las críticas principales a esta literatura han sido que, primero, si bien facilita el análisis de las implicaciones de la política migratoria y la reforma para que las personas inmigrantes tengan legalmente acceso a la política social, este último no representa un eje central en esta literatura. Es decir, no ha prestado mucha atención a la constelación de prácticas que se ha propuesto por la literatura de los regímenes de bienestar para explicar diferentes dimensiones de la migración y la integración. Segundo, esta literatura, al igual que la del Norte, ha mantenido su enfoque en los criterios de elegibilidad de la política social; es decir, los derechos sociales se han analizado principalmente en términos de derechos formales, sin considerar el acceso real a los servicios (Pribble 2015; Morissens 2008; Morissens y Sainsbury 2005; Sainsbury 1996).

Lo anterior significa un vacío en la literatura sobre las formas concretas en las que los inmigrantes interactúan con la política social, los medios por los cuales los servicios sociales se integran en sus estrategias de bienestar y la forma en que se relacionan la migración y la política social. Si en el Norte es importante diferenciar entre los derechos y el acceso real a los servicios sociales, esta diferenciación es aún más importante en el Sur, porque los mercados laborales son más informales (Barrientos 2004), la capacidad institucional es más débil (Baganha 2002) y los regímenes sociales son menos incluyentes y más estratificados (Noy 2013; Martínez-Franzoni 2008; Barba 2007).

Más recientemente, ante estos desafíos en la literatura latinoamericana, se han dado acercamientos más sistemáticos sobre la interacción entre la migración y la política social. Noy y Voorend (2016) comparan los derechos sociales de migrantes en Argentina, Chile y Costa Rica. Se enfocan en el sector de salud. Concluyen que la variación de la extensión de derechos sociales 
depende de la interacción entre la política migratoria y la política social, la estructura y organización del sistema de salud y, dependiendo del contexto, en diferentes grados de los marcos normativos regionales e internacionales.

Voorend (2019) analiza la interacción entre la inmigración y la política social. De manera específica, la pesquisa se centró en el seguro médico público y en la prestación y el acceso a servicios de la salud pública en Costa Rica. Este análisis detalla caso costarricense, su política migratoria y las implicaciones para el acceso a la salud pública de migrantes, así como las formas de protección social más informales de personas inmigrantes en el país. Concluye que la mayor informalidad de los mercados laborales en el Sur, con acuerdos de bienestar generalmente más débiles y peores condiciones de trabajo en comparación con el Norte global, provocan la existencia de más vulnerabilidades para la población inmigrante. Con políticas sociales poco exhaustivas e incapaces de cubrir la totalidad de las poblaciones, la presión por ampliar la cobertura a poblaciones inmigrantes es muy reducida, incluso cuando la política social se basa en el principio del universalismo.

En este sentido, el caso de Costa Rica corrobora lo que Leerkes (2016) establece en el caso de Holanda: su argumento central de que existe una creciente diferenciación en la ciudadanía entre los inmigrantes admitidos formalmente y los que se consideran como "no miembros" o "ilegales". Incluso, se crean diferencias en torno al tipo de estrategias de acceso a protección social informal, que emplean personas inmigrantes ante la dificultad de acceso a los servicios sociales. Al mismo tiempo, esta investigación cuestiona la literatura sobre migración y ciudadanía en la que se afirma que los derechos de los inmigrantes se asemejan cada vez más a los derechos de los ciudadanos nacionales, pues argumenta que esto no parece ser representativo para el Sur.

Este tipo de trabajos se tornan necesarios para ampliar la agenda sobre el nexo entre migración y política pública en América Latina. Sin embargo, el trabajo de Noy y Voorend (2016) se enfoca en derechos sociales, no en acceso de facto a servicios. Asimismo, el trabajo de Voorend (2019) no incorpora un lente comparativo. El trabajo de Leerkes (2016) incorpora el elemento de la informalidad sin ahondar en sus implicaciones para la protección transnacional. Además, ninguno de estos trabajos logra escapar lo que se ha denominado como el nacionalismo metodológico (Levitt 2012); es decir, no reconocen que muchas de las prácticas de protección social pueden transcender las fronteras del Estado.

\section{Transnacionalismo, familias transnacionales y el cuidado}

El desarrollo literario sobre regímenes de bienestar ofrece un marco conceptual-analítico muy sugerente. Se enfoca en las constelaciones de prácticas entre Estado, mercado y familia para estudiar la protección social. Sin embar- 
go, hasta el momento, ha ignorado, casi por completo, a las poblaciones migrantes y carece de un lente transnacional para explicar las dinámicas de protección, que transcienden las fronteras del Estado-nación. Similarmente, la literatura sobre la migración internacional ha tratado el fenómeno migratorio de una miríada de dimensiones, pero no ha incorporado un lente sistemático y comparativo desde la política social, los regímenes de bienestar o, de manera más general, de la protección social.

En ese sentido, la literatura sobre el transnacionalismo surge como la llamada a contener herramientas útiles para estudiar la protección social transnacional, pero esta es una literatura que carece, con unas notables excepciones (Speroni 2019), de insumos de carácter empírico, especialmente para el llamado Sur global (Yeates 2006; Holzmann, Koettl y Chernetsky 2005).

En primer lugar, vale rescatar la definición conceptual del mismo transnacionalismo, que se refiere a procesos económicos, políticos y culturales que se extienden más allá de las fronteras nacionales. En esa línea, es importante acotar que tanto el Estado, los mercados o las familias pueden operar transnacionalmente; es decir, al trascender las fronteras del Estado-nación (Levitt et al. 2016; Faist et al. 2015). Estas dinámicas son sumamente importantes de reconocer con el fin de ampliar su estudio. Por ello, en el presente escrito, se centra esencialmente la atención en el tema de la protección social transnacional y los accesos de las personas migrantes a regímenes de bienestar en espacios transnacionales. Sin embargo, es evidente que los estudios de transnacionalismo pueden abordar un sinfín de temas.

Un enfoque, que ha vinculado la protección social formal con el transnacionalismo, es el de la portabilidad de derechos. Taha $(2013,5-6)$ define este concepto como "la habilidad de los trabajadores migrantes de preservar, mantener y transferir beneficios de un programa de un país a otro y entre localidades de un mismo país, entre diferentes empleos y para distintos miembros de sus hogares". Para algunos migrantes, los planes de pensiones son portátiles y cubren la provisión para la vejez a través de las fronteras; por ejemplo, los ciudadanos estadounidenses que se alojan tras su pensión en países latinoamericanos. En cambio, para otros, como el migrante centroamericano, que partió en búsqueda de su sueño americano, su contribución de años en los Estados Unidos se pierde en caso de que decida regresar a su país de origen.

En ese sentido, los Estados tienen un peso importante en las dinámicas transnacionales, porque habilitan o no la capacidad de que las personas que viven en estos espacios puedan mantener y confirmar sus derechos sociales (Quintero Lima 2012; Holzmann, Koettl y Chernetsky 2005). Ahora bien, ante la ausencia de estas habilitaciones estatales, en muchas ocasiones, las personas migrantes tornan su mirada hacia apoyos de diversa naturaleza para velar por su sustento. Tal es el caso de los migrantes que obtienen medicamentos importados de su país de origen, ya sea de familiares o en el mercado informal (Voorend 2019). O bien, del mismo modo, los flujos de remesas internacionales, que se gastan en vivienda, educación y salud privada, son 
formas de protección social transnacional, que no pasan por la protección social formal estatal.

Para el estudio de la protección social transnacional, la literatura sobre las familias transnacionales, y en particular la que se enfoca en el cuidado, se torna central. La investigación sobre familias transnacionales se centra en las estrategias, en muchos casos, transnacionales, que crean redes familiares para criar y cuidar a sus hijos (Bilecen y Sienkiewicz 2015), a las personas adultas mayores (Zechner 2008) e inclusive a dependientes con estatus migratorio irregular (Parella y Speroni 2018), a través de las fronteras, al utilizar redes tanto formales como informales.

En este sentido, se ha dado un reconocimiento en la literatura a la importancia del cuidado como una práctica de protección social, informal y transnacional. Speroni (2019) presenta un excelente análisis sobre los aportes de esta literatura, que principalmente se divide en tres perspectivas complementarias. Primero, la de las cadenas globales del cuidado que enfatiza en la organización económica de tareas y trabajos remunerados o no del cuidado personal. Esta literatura se enfoca en la división internacional del trabajo reproductivo con énfasis en el encadenamiento transnacional entre trabajos de cuidado remunerados y no remunerados (Carrasco 2011; Orozco 2010).

Segundo, la perspectiva sobre la organización social del cuidado analiza la dimensión política del cuidado o el conjunto de relaciones constituidas a partir de la interacción entre el Estado, el mercado, la familia y el tercer sector o comunidad. Este cuerpo estudia los interfaces entre la política social y la configuración de las dinámicas económicas del cuidado (Speroni 2019; Gonzálvez 2016; Leiva 2015; Orozco 2010).

Finalmente, la perspectiva de la circulación del cuidado se enfoca en las dinámicas sociales de este, sea presencial o a distancia e incluyendo tanto los aspectos económicos, como también los relacionados con lo emocional, del cuidado a niños, personas enfermas y ancianas. Mediante los arreglos familiares transnacionales de distribución y circulación de derechos y obligaciones estudian las redes transnacionales de intercambio recíproco y asimétrico del cuidado (Speroni 2019; Merla y Baldassar 2016; Merla 2014)

Sin embargo, una crítica importante a esta literatura (Speroni 2019; Barglowski et al. 2015) consiste es que en estos estudios se privilegia el tema del cuido familiar y, si bien eso es sumamente importante, no constituye la plenitud de los aspectos que engloban la protección social transnacional que incluye pensión, salud, empleo, educación, comunicación y otros recursos. Varios de estos elementos pueden desarrollarse en espacios transnacionales. En este sentido, el transnacionalismo debe ser incorporado en el estudio de la protección social transnacional no como una categoría de análisis independiente, sino como un lente analítico transversal en reconocimiento del hecho de que las prácticas de protección social (estatal, mercantil, familiar) que podrían o no cruzar esas fronteras. 


\section{De la protección social transnacional a su estudio en el Sur}

De especial interés, para el estudio de la protección social de personas migrantes, es la literatura creciente sobre la protección social transnacional. Ante la falta de un cuerpo de literatura latinoamericano, con notables excepciones como Salazar y Voorend (2019) y Speroni (2019), se discute la literatura que se ha generado en Europa principalmente y, en menor medida, en Estados Unidos (Parella y Speroni 2018; Dobbs y Levitt 2017; Faist 2017; Gabarot 2016; Levitt et al. 2016; Faist et al. 2015; Avato, Koettl y SabatesWheeler 2010; Koettl y Chernetsky 2005). Esta literatura, hasta ahora más conceptual que empírica, de nuevo con notables excepciones como en Faist y Bilecen (2015), genera una serie de interrogantes en torno a la protección social de migrantes, que abren un campo investigativo amplio y rico en su abordaje y posibles tipos de enfoque.

¿Cómo y en qué medida son protegidas estas personas que se mueven entre diferentes Estados? ¿Cuáles formas institucionales, mercantiles, del tercer sector y familiares se generan ante la necesidad de migrantes (y sus familias) de lidiar con los riesgos sociales de la vida? ¿Cuán formales o informales son estas prácticas? ¿Cuáles de estas prácticas trascienden las fronteras del Estado-nación? Es decir, ¿cuáles de estas prácticas obtienen un carácter transnacional?

Sin embargo, la literatura todavía encuentra el desafío de dar respuesta a muchas de estas preguntas, sobre todo, con respaldo empírico. Lo que sí ofrece son reflexiones sobre la importancia de desarrollar contenido sobre esta agenda de trabajo académico (Yeates 2006; Smith y Bailey 2004) y propuestas conceptuales y metodológicas de cómo se debe abordar la protección social transnacional (Levitt et al. 2016; Bilecen y Sienkiewicz 2015; Faist et al. 2015).

En esta literatura, no existe todavía un consenso irrefutable sobre el propio concepto de protección social transnacional. Se trata de un área pendiente, para la cual destaca la propuesta de Levitt et al. $(2016,6)$, quienes definen la protección social transnacional como "las políticas, programas, personas, organizaciones e instituciones que proporcionan y protegen a las personas en diferentes áreas relacionadas al bienestar (cuidado, pensiones, incapacidad, salud, familia, mercado laboral, desempleo, vivienda, educación) de manera transnacional".

A partir de tal definición, es necesario recalcar que existe un reconocimiento por parte de esta literatura de que las personas migrantes se mueven entre espacios con distintas capacidades estatales, donde el alcance de la protección social formal puede variar considerablemente. Esto provoca que la protección social de estas personas se compone de una combinación de su acceso a instituciones formales e informales en los países emisores y receptores de este grupo poblacional migrante (Faist et al. 2015). 
De tal forma, su idea central radica en que las personas migrantes recién llegadas a nuevos países se encuentran en una posición de suma vulnerabilidad, donde están alejados de sus comunidades y no cuentan con acceso a importantes redes de apoyo informales (familiares, amistades) (Levitt et al. 2016; Barglowski, Bilecen y Amelina 2015; Bilecen y Sienkiewicz 2015; Zechner 2008). Al mismo tiempo, observan que la protección ofrecida por el Estado al que arriban, usualmente, les implica una serie de restricciones que pasa por elementos como el estatus de residencia, la estabilidad laboral y un cierto ingreso económico periódico (Fortuny y Chaudry 2011). Esto, normalmente, los deja en una posición desventajosa para garantizar su bienestar personal y les deja en desamparo de protección social en muchas ocasiones (Levitt et al. 2016; Faist 2008; Faist 2000).

Para las personas migrantes, que se trasladan a Estados con regímenes de protección social formal fuertes en el Norte global, el estatus migratorio de residencia y la ciudadanía influyen fuertemente en sus derechos en el país anfitrión. También, puede variar considerablemente entre diferentes jurisdicciones subnacionales, tal como en el interior de los diferentes Estados que conforman países como Estados Unidos o España (Dobbs y Levitt 2017; Moreno Fuentes 2015; Quintero Lima 2012).

Fuera del Norte global, el Estado nacional y el Estado de derecho tienden a estar menos firmemente establecidos. Si bien los factores que determinan el acceso a la protección social son similares, la informalidad juega un papel más importante. En otras palabras, la protección social formal (estatal) tiene menos predominancia de manera general, lo que implica que formas de protección social informal (familia, mercado informal, etc.) toman más centralidad. Esto más aun para personas migrantes, que tienden a construir prácticas de protección social que trasciende las fronteras nacionales. En ambos casos, las personas sin estatus migratorio legal o residencial, son particularmente vulnerables, porque su acceso a las instituciones públicas de protección social es especialmente limitado (Avato, Koettl y Sabates-Wheeler 2010).

Por ello, un mensaje importante de la literatura sobre protección social transnacional consiste en que se torna clave dirigir la atención a las prácticas tanto formales como informales de protección social. Se trasciende en el análisis del umbral del Estado únicamente. Un marco de estudio ha sido catalogado bajo diversos nombres. Para Levitt et al. (2016), se le puede llamar el "entorno de recursos" (resource environment), mientras Faist (2014) lo denomina "ámbitos de protección" (realms of protection). Curiosamente, la conceptualización es muy similar al concepto de "mundos de bienestar" (Martínez-Franzoni 2008), que propone la literatura de los regímenes de bienestar.

Sin embargo, más allá de su etiqueta, este entorno de recursos o ámbitos de protección de una persona está constituido por la combinación de todas las potenciales protecciones disponibles, que se pueden categorizar de cuatro posibles fuentes: Estados, mercados, el tercer sector y redes sociales. EI conjunto de protecciones, que esté finalmente disponible, depende de la for- 
taleza y la capacidad de los Estados emisores y receptores, la naturaleza del acceso a los mercados en ambos lados de la frontera, la ecología organizacional del tercer sector (es decir, el número y tipos de organizaciones, lo que hacen y su capacidad de provisionar servicios) y las características de las personas migrantes individuales y sus familias y comunidades.

Ahora bien, un último elemento clave por rescatar de esta literatura. Ella aboga, sin excepciones, por la importancia de la interseccionalidad en el análisis de la protección social transnacional (Faist 2017; Levitt et al. 2016; Barglowski, Bilecen y Amelina 2015; Bilecen y Sienkiewicz 2015; Sienkiewicz, Sadovskaya y Amelina 2015; Faist 2014). Existe claridad de que las prácticas de protección social, sean estas transnacionales o no, son directamente influenciadas por factores relacionados con clase, género, etnia, discapacidades, entre otros.

Por ejemplo, el acceso a un seguro médico privado tiende a ser más accesible para alguien de un estrato socioeconómico más alto que para una persona que gana el salario mínimo. Las mujeres tienen otro tipo de acceso a la política social y normalmente tienden encargarse de la organización del cuido no remunerado. Ciertas poblaciones migrantes tienden a ser excluidas de ciertos servicios sociales por procesos de etnicización, xenofobia y discriminación. Así, la literatura reconoce que las prácticas de protección social se construyen en un contexto de relaciones sociales y, por ende, de relaciones de poder.

\section{Puntos de acuerdo para un marco analítico desde el Sur Global}

La literatura, principalmente del Norte global sobre la protección social transnacional, ofrece un conjunto de elementos diversos que sirven para pensar la protección social en y desde el Sur, ante el actual reto de generar información sobre las dinámicas de protección social formal e informal de migrantes en estos países del mundo (Salazar y Voorend 2019; Speroni 2019; Yeates 2006; Holzmann, Koettl y Chernetsky 2005).

El desafío, por tanto, es ampliar las discusiones en torno a la protección social transnacional de migrantes, que se mueven entre países del Sur, caracterizados por ser grandes naciones emisoras de migrantes y, a su vez, también receptoras de estos. Introducir un lente transnacional es un reconocimiento importante de la variedad de las prácticas de protección social de personas migrantes. Esto permitiría atender dos retos importantes. Primero, impulsar estudios comparativos de las políticas y otras protecciones sociales informales, que se enfocan en poblaciones migrantes. Segundo, trascender el nacionalismo metodológico al dirigir la mirada a las dinámicas entre el país de origen y el de destino ( $y$ todo lo que pasa en el medio). Esto no solo genera un aporte al conocimiento de cómo y en qué medida las personas migran- 
tes acceden a diferentes constelaciones de protecciones sociales, sino que también permite entender las dinámicas de los Estados, sus regímenes de protección social y las relaciones transnacionales a las que estos regímenes dan lugar.

Para fortalecer esta línea de investigación, se presentan algunos acuerdos básicos iniciales para el estudio de la protección social transnacional, que encuentran su origen en los cuerpos de literatura previamente referenciados. Corresponden a elementos críticos que pueden ser subrayados tras el repaso realizado por la diferente literatura sobre esta temática.

Primero, el concepto de protección social incorpora más que la protección social estatal, es decir, la política social. Tanto la literatura de regímenes de bienestar y la de protección social transnacional enfatizan en la necesidad de trascender el Estado al incorporar otras dimensiones y actores que proveen protección social.

Segundo, al retomar los mismos cuerpos de literatura, destaca la importancia de incorporar al análisis de la protección social la dimensión mercantil y la dimensión informal constituida por las familias, las redes sociales de conocidos y amistades y la comunidad de manera más amplia. A la informalidad, en esta literatura creciente, debe otorgársele su peso analítico para poder captar esos entornos de recursos que tienen las personas migrantes a su disposición para su protección social en un contexto particular. En términos prácticos, esto implicaría estudios que transcienden las fronteras del Estado, con investigaciones que usen métodos mixtos que permiten capturar la riqueza de diferentes experiencias particulares, en combinación con datos más generalizables.

Tercero, destaca la importancia en el análisis de la protección social formal de enfocarse no solo en los derechos sociales, los criterios de elegibilidad y los requisitos para tener acceso a los servicios sociales, sino también es crucial analizar el acceso real de las personas migrantes con respecto de los servicios sociales, la suficiencia que le generan estos servicios a las personas para lidiar con los riesgos sociales de la vida y los costos que implican. Existen nuevas agendas de investigación (véase por ejemplo: Niedzwiecki y Voorend 2019; Voorend 2019) que demuestran que la brecha entre compromisos estatales con derechos sociales y el acceso real es causada por diferentes barreras de índole legal, institucional, de facto y de agencia. Dependiendo del acceso real, pueden surgir nuevas y más complejas formas de protección social informal y transnacional.

Finalmente, se enfatiza la necesidad de incorporar el lente de la interseccionalidad en el análisis de la protección social, en reconocimiento que las prácticas y los niveles de protección están entrelazados con clase, género, etnia/ raza. En particular, el estudio de las barreras a las diferentes formas de protección que enfrentan poblaciones migrantes debe reconocer que existen sistemas de desventaja o discriminación superpuestos e interdependientes. 
Con esto, y reiterando la concepción de la protección social, como las políticas, los programas, las personas, las organizaciones e instituciones que proveen y protegen a las personas (Levitt et al. 2016), se entiende lo último como la capacidad de lidiar con riesgos sociales (Martínez-Franzoni 2008). Se presenta una primera propuesta de un marco analítico para el estudio de la protección social de migrantes. Para ayudar en esta tarea, en la Figura 1, se presenta visualmente la constelación de diferentes prácticas de protección social, en un entorno de recursos de una persona migrante de su país de origen Y, quien reside en el país X. Esta figura se basa en Levitt et al. (2016) e incorpora distintos elementos analíticos:

Figura 1. Entorno de recursos de una persona migrante

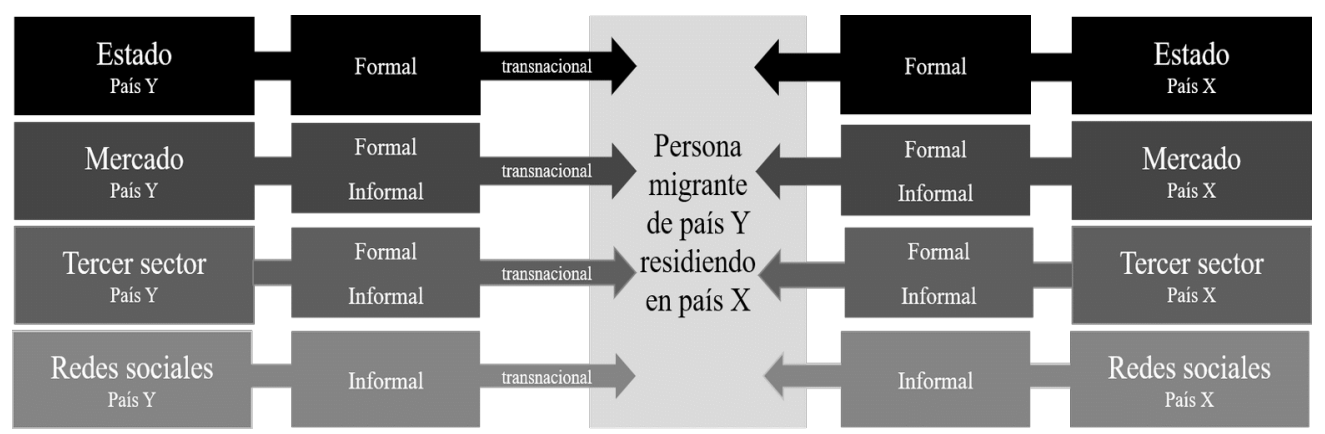

Fuente: Elaboración propia con base en Levitt, Lloyd, Mueller y Viterna (2016).

Interesa analizar las flechas, que corresponden a las prácticas de protección social dentro de esa constelación que se puede llamar "entorno de recursos" (Levitt et al. 2016). Es importante anotar que, si bien la figura muestra todas las opciones de diferentes prácticas, en la realidad una persona migrante no siempre va a tener acceso a todas estas prácticas. Es más, es muy poco probable que lo tenga. Es decir, la primera tarea investigativa es mapear cuáles flechas existen para cada caso, según sus condiciones particulares.

Segundo, las flechas pueden variar en su tamaño. En otras palabras, las diferentes prácticas de protección social pueden habilitar mayores o menores niveles de protección. Por lo tanto, después de establecer cuáles prácticas están disponibles en el entorno de recursos de la persona inmigrante, es importante analizar el nivel de la protección real que ofrecen estas prácticas. Tercero, resulta esencial contemplar cuáles de estas prácticas tienen un carácter transnacional y cuáles son las estrategias por tomar cuando una persona no tiene acceso algunas de estas prácticas, en especial a la protección social estatal (formal).

Se propone situar, en un plano central, a la persona inmigrante. La variable dependiente es la protección social en el sentido más amplio, sea esta formal o informal, con el objetivo principal de caracterizar los entornos de recursos para evaluar la capacidad de lidiar con riesgos sociales. La protección social 
estatal (política social: acceso, calidad, suficiencia, costo) juega un papel clave en estos entornos de recursos como variable independiente. Al mismo tiempo, interesan las otras prácticas de protección social mercantiles, del tercer sector (iglesias en ambos países, organizaciones proderechos inmigrantes, asociaciones de migrantes) y de redes comunitarias y familiares como posibles alternativas -y como otras variables independientes- distintas a la protección social estatal.

Finalmente, interesa adicionalmente comprender la variación en el acceso. ¿Por qué una persona migrante logra acceso a la protección social estatal, mientras que otra no y debe recurrir a protección social mediante redes familiares y comunitarias? Para esto, se sugiere adicionar al marco analítico, las barreras a la inclusión siguiendo a Fischer (2011), que se entienden como procesos de obstrucción y no como un resultado (de ser o no excluido). La adaptación consiste en que se usa la palabra barreras, en el entendido que estas dificultan, pero no necesariamente imposibilitan, el acceso a la protección social. Dichas barreras se pueden generar por medio de factores estructurales, mecanismos legales e institucionales o representaciones deliberadas de actores individuales (Fischer 2011). Esta definición es útil para estudiar la exclusión de los inmigrantes y la política de migración, porque trasciende el análisis de un Estado o del resultado de una exclusión; antes bien, se concentra en los mecanismos que causan el proceso de exclusión.

De tal manera, el marco analítico propuesto ofrece una perspectiva general del entorno de recursos mediante los que personas migrantes pueden acceder o no la seguridad social formal o informal. Contemplar tanto las diferentes fuentes de esa protección social, como las diversas barreras que puedan presentarse para acceder a estas, evidencia las constelaciones de factores que pueden darse en los distintos casos.

La población migrante tiene así distintos escenarios en los cuáles puede posicionarse, según factores y condiciones en las que realiza su nueva vida en el país de destino al que llega. Los elementos conceptuales, que usualmente la literatura aborda de una forma preconcebida, como la ciudadanía o la informalidad, deben ser repensados en aras de poder dar una comprensión mayor a la cobertura de la vulnerabilidad social de estas personas. En este artículo subrayamos la importancia de ir avanzando en la investigación de estas constelaciones en aras de generar categorías analíticas y determinar patrones que permitan caracterizar la protección social transnacional que se da en un Sur global.

\section{Conclusión}

El estudio de la protección social transnacional formal e informal es un tema creciente en la academia. Sin embargo, ante los flujos migratorios que se amplifican año tras año, y los retos que esto implica para Estados en cuanto a la incorporación de poblaciones inmigrantes en políticas (sociales) y merca- 
dos laborales, se amerita una agenda de investigación desde el Sur global que incorpore amplios y diversos estudios empíricos y discusiones analíticas para entender en qué medida y cómo se protegen a personas migrantes en diferentes etapas de su proceso migratorio.

Como se buscó evidenciar en el artículo, ya existen aportes importantes de distintas corrientes de literatura que vienen a nutrir los elementos que engloban los recursos, las barreras y los elementos que rodean el acceso de las personas migrantes a los sistemas formales de políticas, servicios sociales y fuentes alternativas, muchas veces informales, de protección social. Sin embargo, no solo urge una agenda de investigación sobre y desde el Sur global al enfatizar en la no tan frecuente capacidad estatal de ofrecer protección social formal y la entonces más frecuente informalidad de las constelaciones de protección social que surgen como alternativas, sino también urge la aplicación de un lente transnacional para entender cómo se organiza la protección social en las vidas transnacionales de las personas migrantes.

Siguiendo propuestas conceptuales como la de Levitt et al. (2016), en este artículo, se propone un posible marco analítico para empezar esta tarea a partir de la cual pueden surgir numerosas líneas de investigación y análisis, que conjuguen elementos de las diferentes literaturas.

El análisis se debe enfocar en las dinámicas internas tanto de los países receptores como emisores de migrantes y sus sistemas formales de protección social, los alcances de estos para cubrir población nacional y migrante, las formas de protección social informales y transnacionales que surgen ante sus vacíos y el rol del tercer sector (iglesias, ONGS, etc.) y de las redes sociales y familiares que cruzan las fronteras nacionales.

En particular, interesa reconstruir los entornos de recursos de las distintas personas inmigrantes y las barreras al acceso a la protección social formal, que podrían implicar los diferentes contextos migratorios. Así, la protección social transnacional se convierte en un tema medular para entender los contextos y las dinámicas migratorios que hoy, más que nunca, desafían al Sur a producir conocimiento acerca de estas realidades que engloban a millones de personas.

\section{Bibliografía}

Arza, Camila. 2009. «Back to the State: Pension Fund Nationalization in Argentina». UNRISD Conference Social and Political Dimensions of the Global Crisis: Implications for Developing Countries. Geneva, 12-13 de noviembre de 2009. 
Asa, Pablo y Pablo Ceriani. 2005. «Los acuerdos del Mercosur y la nueva ley de migraciones en Argentina». Revista Beyond Law 11(28): 39-65.

Avato, Johanna, Johannes Koettl y Rachel Sabates-Wheeler. 2010. «Social Security Regimes, Global Estimates, and Good Practices: The Status of Social Protection for International Migrants». Revista World Development 38(4): 455-466. doi:10.1016/j.worlddev.2009.10.003.

Baganha, Maria. 2002. «Immigrants' social citizenship and labour market dynamics in Portugal». En: Inmigration and Welfare: Challenging the Borders of the Welfare State. Editado por Michael Bommes y Andrew Geddes. London: Routhledge.

Baldwin-Edwards, Martin. 2002. «Immigration and the Welfare State: A European Challenge to American Mythology». MMO Working Paper No. 4, November of 2002.

Barba, Carlos. 2007. «América Latina: regímenes de bienestar en transición durante los años noventa». Cuaderno CRH 20(50): 197-211. doi:10.1590/S0103-49792007000200002.

Barglowski, Karolina, Başak Bilecen y Anna Amelina. 2015. «Approaching Transnational Social Protection: Methodological Challenges and Empirical Applications». Population, Space and Place 21(3): 215-26. doi:10.1002/psp.1935.

Barrientos, Armando. 2004. «Latin America: towards a liberal-informal welfare regime? ». En Insecurity and Welfare Regimes in Asia, Africa, and Latin America: Social policy in development contexts. Editado por lan Gough y Geoffrey D. Wood. Cambridge, UK, New York: Cambridge University Press.

Begala, Silvana. 2012. «El reconocimiento diferenciado de derechos: primer obstáculo al acceso a la justicia de las personas migrantes». Revista Derecho y Ciencias Sociales 6: 3-24. 
Bilecen, Başak y Karolina Barglowski. 2015. «On the Assemblages of Informal and Formal Transnational Social Protection». Population, Space and Place 21(3): 203-14. doi:10.1002/psp.1897.

Bilecen, Başak y Joanna Sienkiewicz. 2015. «Informal Social Protection Networks of Migrants: Typical Patterns in Different Transnational Social Spaces». Population, Space and Place 21(3): 227-43. doi:10.1002/psp.1906.

Bommes, Michael y Geddes, Andrew. 2000. Immigration and Welfare. London, Routledge.

Carrasco, Cristina. 2011. "La economía del cuidado: planteamiento actual y desafíos pendientes». Revista de Economía Crítica 11.

Carrasco, Lorena. 2008. "Living on the Margins: illness and healthcare among Peruvian migrants in Chile». Tesis doctoral. Leiden University.

Castles, Stephen y Mark Miller. 2009. The Age of Migration: International Population Movements in the Modern World. Palgrave Macmillan.

Centro de Estudios Legales y Sociales - CELS. 2013. Migrantes. Buenos Aires.

Ceriani, Pablo. 2011. «Luces y sombras en la legislación migratoria latinoamericana.». Nueva Sociedad, 233: 68-86.

Cerutti, Marcela. 2011. Salud y migración internacional: mujeres bolivianas en la Argentina. Buenos Aires: Programa Naciones Unidas para el Desarrollo-PNUD; Centro de Estudios de Población-CENEP.

Courtis, Corina, Gabriela Liguori y Marcela Cerutti. 2010. Migración y Salud en Zonas Fronterizas: el Estado Plurinacional de Bolivia y la Argentina. Santiago, Chile.

Courtis, Corina y María Pacecca. 2007. «Migración y derechos humanos: una aproximación crítica al "nuevo paradigma" para el tratamiento de la 
cuestión migratoria en la Argentina». Revista Jurídica de Buenos Aires. Edición especial sobre Derechos Humanos: 183-200.

Department of Economic and Social Affairs. 2019. «International Migrant Stock 2019».

Dobbs, Erica y Peggy Levitt. 2017. «The missing link? The role of subnational governance in transnational social protections». Oxford Development Studies 45, 1: 47-63. doi:10.1080/13600818.2016.1271867.

Domenech, Eduardo. 2007. «La agenda política sobre migraciones en América del sur: el caso de la Argentina». Revue Européenne des Migrations Internationales 23: 71-94.

Domenech, Eduardo. 2008. «La ciudadanización de la política migratoria en la región sudamericana: vicisitudes de la agenda global». En Las migraciones en América Latina, editado por Susana Novick. CLACSO. Buenos Aires.

Domenech, Eduardo. 2011. «Crónica de una "amenaza" anunciada. Inmigración e 'ilegalidad': visiones de Estado en la Argentina contemporánea». En La Construcción del sujeto migrante en América Latina: Prácticas, representaciones y categorías. Editado por Bela Feldman-Bianco, Liliana Rivera-Sánchez, Carolina Stefoni y Marta Villa. Quito, Ecuador.

Esping-Andersen, Gøsta. 1990. The Three Worlds of Welfare Capitalism. Princeton N.J.: Princeton University Press.

Faist, Thomas. 2000. «Transnationalization in international migration: implications for the study of citizenship and culture». Ethnic and Racial Studies 23(2): 189-222. doi:10.1080/014198700329024.

Faist, Thomas. 2008. «Migrants as transnational development agents: an inquiry into the newest round of the migration-development nexus». Population, Space and Place 14(1): 21-42. doi:10.1002/psp.471. 
Faist, Thomas. 2013. «Transnational Social Protection: An Emerging Field of Study». Center on Migration, Citizenship and Development, no.113.

Faist, Thomas. 2014. «On the transnational social question: How social inequalities are reproduced in Europe». Journal of European Social Policy 24(3): 207-22. doi:10.1177/0958928714525814.

Faist, Thomas. 2017. «Transnational social protection in Europe: a social inequality perspective». Oxford Development Studies 45(1): 20-32. doi:10.1080/13600818.2016.1193128.

Faist, Thomas y Başak Bilecen. 2015. «Social Inequalities Through the Lens of Social Protection: Notes on the Transnational Social Question». Population, Space and Place 21(3): 282-93. doi:10.1002/psp.1879.

Faist, Thomas, Başak Bilecen, Karolina Barglowski y Joanna J. Sienkiewicz. 2015. «Transnational Social Protection: Migrants' Strategies and Patterns of Inequalities». Population, Space and Place 21: 193-202.

Filgueira, Fernando. 2007. «Latin American Social States: Critical Junctures and Critical Choices». En Democracy and Social Policy, editado por Yusuf Bangura. Basingstoke: Palgrave Macmillan.

Filgueira, Fernando, Luis Reygadas, Juan P. Luna y Pablo Alegre. 2011. «Shallow States, Deep Inequalities, and the Limits of Conservative Modernization: The Politics and Policies of Incorporation in Latin America». En The Great Gap: Inequality and the Politics of Redistribution in Latin America, editado por Merike Blofield, 243-77. University Park, Pa.: Pennsylvania State University Press.

Fischer, Andrew. 2010. «Towards Genuine Universalism within Contemporary Development Policy». IDS Bulletin 41(1).

Fischer, Andrew. 2011. «Reconceiving social exclusion». BWPI Working Paper 146.

Fortuny, Karina y Ajay Chaudry. 2011. A Comprehensive Review of Immigrant Access to Health and Human Services. The Urban Institute. 
Gabarot, Mariana. 2016. «Familias transnacionales y política social en las comunidades de origen: una visión cualitativa de la relación entre migración y desarrollo». Migración y Desarrollo 14(27): 139-65.

Giustiniani, Ruben. 2004. Migración: un derecho humano. Buenos Aires: Prometeo.

Gonzálvez, Herminia. 2016. «Los cuidados en la migración transnacional.» Revista Sur 13(24).

Gough, lan y Geof Wood. 2004. Insecurity and Welfare Regimes in Asia, Africa, and Latin America: Social policy in development contexts. Cambridge, UK, New York: Cambridge University Press.

Haggard, Stephan y Robert Kaufman. 2008. Development, Democracy, and Welfare States: Latin America, East Asia, and Eastern Europe. Princeton: Princeton University Press.

Holzmann, Robert, Johannes Koettl y Taras Chernetsky. 2005. «Portability Regimes of Pension and Health Care Benefits for International Migrants: An Analysis of Issues and Good Practices». Social Protection 0519: 3-63. World Bank.

Huber, Evelyne y John Stephens. 2012. Democracy and the Left: Social Policy and Inequality in Latin America. Chicago: The University of Chicago Press.

Jelin, Elizabeth. 2006. Salud y migración regional. Ciudadanía, discriminación y comunicación intercultural. Buenos Aires: IDES.

Joppke, Christian. 2012. «Citizenship and Immigration». Ethnicities 12(6): 4463. doi:10.1177/1468796812449706.

Kivisto, Peter y Thomas Faist. 2007. Citizenship: Discourse, theory, and transnational prospects. Malden MA: Blackwell Pub. 
Kofman, Eleonore, Annie Phizacklea, Parvati Raghuram y Rosemary Sales. 2000. Gender and International Migration in Europe: Employment, Welfare and Politics. Florence: Taylor and Francis.

Leerkes, Arjen. 2016. «Back to the poorhouse? Social protection and social control of unauthorised immigrants in the shadow of the welfare state». Journal of European Social Policy 26 (2): 140-54. doi:10.1177/0958928716637139.

Leiva, Sandra. 2015. «Organización social del cuidado en Bolivia y Chile: Estado y cuidadanía». Revista Austral de Ciencias Sociales 28: 61-81. doi:10.4206/racs.2015.n28-04.

Levitt, Peggy. 2012. «What's wrong with migration scholarship? A critique and a way forward». Identities 19(4): 493-500. doi:10.1080/1070289X.2012.676255.

Levitt, Peggy, Charlotte Lloyd, Armin Mueller y Jocelyn Viterna. 2016. «Transnational social protection: setting the agenda». Oxford Development Studies 45(1): 2-19. doi:10.1080/13600818.2016.1239702.

Lucassen, Leo. 2016. Migration, Membership Regimes and Social Policies: $A$ View from Global History. Northampton: Edward Elgar Publishing.

Martínez-Franzoni, Juliana. 2008. «Welfare Regimes in Latin America: Capturing Constellations of Markets, Families and Policies». Latin American Politics and Society 50(2): 67-100.

Martínez-Franzoni, Juliana y Diego Sánchez-Ancochea. 2016. The Quest for Universal Social Policy in the South: Actors, Ideas and Architectures. New York: Cambridge University Press.

Merla, Laura. 2014. «La circulación de cuidados en las familias transnacionales / The circulation of care in transnational families» Revista CIDOB d'Afers Internacionals 106-107: 85-104. 
Merla, Laura y Loretta Baldassar. 2016. «Response: Special Review Symposium, Transnational Families, Migration and the Circulation of Care». Papers: revista de sociología 101(2): 275-84.

Mkandawire, Thandika. 2005. «Targeting and Universalism in Poverty Reduction». Social Policy and Development 23: 1-18.

Moreno Fuentes, Francisco. 2015. «El puzzle de la exclusión sanitaria de los inmigrantes indocumentados en España». Anuario de la Inmigración en España: 278-99.

Morissens, Ann. 2008. Migration, welfare states and the incorporation of migrants in different welfare regimes. Geneva: UNRISD.

Morissens, Ann y Diane Sainsbury. 2005. «Migrants' Social Rights, Ethnicity and Welfare Regimes». Journal of Social Policy 34(4): 637-60. doi:10.1017/S0047279405009190.

Niedzwiecki, Sara y Koen Voorend. 2019. «Barriers to Social Policy and Immigration in Latin America». Paper prepared for the Fifth Southwest Workshop on Mixed Methods Research. Mexico City, October 23-24.

Novick, Susana. 2008. «Migración y políticas en Argentina: Tres leyes para un país extenso (1876-2004).». Cuadernos del Pensamiento Crítico Latinoamericano. CLACSO. Buenos Aires Argentina.

Novick, Susana. 2010. «Políticas migratorias en la Argentina: experiencias del pasado, reformas actuales y expectativas futuras.». Ponencia Consejo Nacional de Investigaciones Científicas y Técnicas -CONICET. Universidad de Buenos Aires.

Novick, Susana. 2012. «Transformations and Challenges of Argentinean Migratory Policy in Relation to the International Context». Migraciones Internacionales 6(22): 206-39. doi:10.17428/rmi.v6i22.744.

Noy, Shiri. 2013. "Globalization, international financial institutions and health policy reform in Latin America.». Tesis Doctoral. Indiana University. 
Noy, Shiri y Koen Voorend. 2016. "Social Rights and Migrant Realities: Migration Policy Reform and Migrants' Access to Health Care in Costa Rica, Argentina, and Chile». Journal of International Migration and Integration 17(2): 605-29. doi:10.1007/s12134-015-0416-2.

Orloff, Ana. 1993. The Politics of Pensions: A Comparative Analysis of Britain, Canada, and the United States, 1880-1940. Madison.

Orozco, Amaia. 2010. Cadenas globales de cuidado ¿qué derechos para un régimen global de cuidados justos? Santo Domingo: UN-INSTRAW.

Oteiza, Enrique y Susana Novick. 2000. Inmigración y derechos humanos Política y discurso en el tramo final del menemismo. Documento de Trabajo del IIGG.

Papadopulos, Theodoros. 2011. Immigration and the variety of migrant integration regimes in the European Union. Bristol: The Policy Press.

Parella, Sònia y Thales Speroni. 2018. «Las perspectivas transnacionales para el análisis de la protección social en contextos migratorios». Autoctonía. Revista de Ciencias Sociales e Historia 2(1): 37-56. doi:10.23854/autoc.v2i1.59.

Pribble, Jennifer. 2015. «The Politics of Building Municipal Institutional Effectiveness in Chile». Latin American Politics and Society 57(3): 100121.

Quintero Lima, María. 2012. «La protección social de los derechos laborales en materia de seguridad y salud: Una asimetria comunitaria y sus remedios internacionales». Cuadernos de Derecho Transnacional 4(1): 180-221.

Rudra, Nita. 2007. "Welfare States in Developing Countries: Unique or Universal?». The Journal of Politics 69(2): 378-96.

Sabates-Wheeler, Rachel y Rayah Feldman. 2011. «Introduction: Mapping Migrant Welfare onto Social Provisioning». En Migration and Social Protection: Claiming Social Rights Beyond Borders 3-35. 
Sainsbury, Diane. 1996. Gender, Equality and Welfare States. Cambridge University Press.

Salazar, Sergio y Koen Voorend. 2019. Protección social transnacional en Centroamérica. Reflexiones a partir de tres contextos de movilidad. Cahiers des Amériques latines.

Schierup, Carl-Ulrik, Peo Hansen y Stephen Castles. 2006. Migration, citizenship, and the European welfare state: A European dilemma. Oxford: Oxford University Press.

Sienkiewicz, Joanna, Yelena Sadovskaya y Anna Amelina. 2015. «The Kazakh-German Social Space: Decreasing Transnational Ties and Symbolic Social Protection». Population, Space and Place 21(3): 27081. doi:10.1002/psp.1905.

Smith, Darren y Adrian Bailey. 2004. «Linking Transnational Migrants and Transnationalism». Population, Sapace and Place 10: 357-60.

Speroni, Thales. 2019. Desencajes y Bricolajes de la Protección Social: Las Familias Transnacionales Bolivianas en Barcelona y Sao Paulo. Tesis doctoral. Universitat Autónoma de Barcelona.

Taha, Nurulsyahirah; Karin Astrid Siegmann y Mahmood Messkoub. 2013. «How portable is social security for migrant workers? A review of literature». International Social Security Review. vol. 68(1) doi:1111/issr.12061

Torres, Amy. 2012. «Universal Health Care in Argentina: Fact or Fiction? An Analysis of Bolivian Immigrant Women's Access to Health Care in Buenos Aires, Argentina.». Tesis de pregrado. Wesleyan University.

van Hooren, Franca. 2011. «Caring Migrants in European Welfare Regimes: The policies and practice of migrant labour filling the gaps in social care». Tesis doctoral. European University Institute. 
Varela, Graciela. 2005. La legislación argentina migratoria. En Migraciones, Globalización y género en Argentina y Chile. Editado por Cecym, Fundación Instituto de la Mujer. Buenos Aires, Argentina.

Voorend, Koen. 2019. ¿Un imán de bienestar en el Sur? Migración y política social en Costa Rica. San José, Costa Rica: Universidad de Costa Rica.

Wilkinson, Mick y Gary Craig. 2011. «Wilful negligence: migration policy, migrants' work and the absence of social protection in the UK». En Migration and welfare in the new Europe: Social protection and the challenges of integration, editado por Emma Carmel, Alfio Cerami y Theodoros Papadopoulos 176-94. Bristol UK: Policy Press.

Yeates, Nicola. 2006. «GSP Forum: New (?) Directions in Global Social Policy». Global Social Policy 6(1): 16-20.

Zechner, Minna. 2008. "Care of older persons in transnational settings». Journal of aging studies 22(1): 32-44. doi:10.1016/j.jaging.2007.02.002. 